\title{
Determination of Volume and Surface Scattering from Saline Ice Using Ice Sheets with Precisely Controlled Roughness Parameters
}

\author{
Jonathan W. Bredow, Member, IEEE, Ronald L. Porco, Adrian K. Fung, Fellow, IEEE, \\ Saibun Tjuatja, Member, IEEE, Kenneth C. Jezek, Sivaprasad Gogineni, and Anthony J. Gow
}

\begin{abstract}
Experiments were performed at the U.S. Army Cold Regions Research and Engineering Laboratory (CRREL) in Hanover, NH, to precisely determine the relative contributions of surface and volume scattering from saline ice that has wellknown surface roughness characteristics. The ice growth phase of the experiment made use of two 6-ft diameter tanks and a 6-ft diameter mold with known roughness statistical parameters of rms height $=0.25 \mathrm{~cm}$ and Gaussian correlation (correlation length $=2.0 \mathrm{~cm}$ ). One tank was used for growing a moderately thick saline ice sheet with very smooth surface, and the other was used for growing a thin layer of freshwater ice over the surface mold. The latter resulted in a layer with one statistically known rough boundary and one smooth boundary. Wide-bandwidth, multiple incidence angle backscattering measurements were performed, first on the bare saline ice sheet and then on the same sheet after the thin freshwater ice sheet was placed on top of it. Results indicate that the surface scattering dominates over saline ice volume scattering at all frequencies for low incidence angles for both the very smooth and Gaussian rough surfaces. The significance of volume scattering depends strongly on angle of incidence, frequency, volume scattering albedo, surface roughness, and surface correlation function.
\end{abstract}

\section{INTRODUCTION}

L ABORATORY measurements of saline and desalinated ice have been ongoing at the US Army Cold Regions Research and Engineering Laboratory (CRREL) for about a decade. The purpose of this program has been to understand and model how electromagnetic waves interact with sea ice. The work reported here was performed as a part of this program and deals with radar measurements: It addresses the question of the relative contributions of volume and surface scattering from saline ice. It is important because it answers these questions with more precision than any other study to date. This is a result of the generation of ice surfaces with known statistical roughness parameters, a measurement setup that facilitates the collection of a large number of

Manuscript received November 2, 1995; revised May 2, 1995. This work was supported by the Office of Naval Research under ONR Grant N0001490-J-1329

J. W. Bredow, R. L. Porco, A. K. Fung, and S. Tjuatja are with the Wave Scattering Research Center, University of Texas at Arlington, Arlington, TX 76019 USA.

K. C. Jezek is with the Byrd Polar Research Center, Ohio State University, Columbus, $\mathrm{OH} 43210$ USA.

S. Gogineni is with Remote Sensing Laboratory, University of Kansas, Lawrence, KS 66045 USA.

A. J. Gow is with Cold Regions Research and Engineering Laboratories, Hanover, NH 03755 USA.

IEEE Log Number 9513903. independent samples, and a very sensitive measurement system that essentially eliminates the effect of antenna beamwidth on measurements.

The effort and the results are presented in four parts. First, a description is given of how the saline ice sheet was grown and how its roughness was generated. Next the measurements are described, including the radar instrument, sampling method, ranges of parameters used, and calibration. Then the factors involved in data analysis are discussed and the results are compared with predictions of a simple scattering model. Finally, a summary of the important findings and a description of anticipated follow-on work is given.

\section{Development of THE SMOOTH AND ROUGH SALINE ICE LAYERS}

Prior to the onset of experiments at CRREL during the winter of 1993-1994 two tanks were fabricated, one for growing a thick $(20 \mathrm{~cm})$ saline ice sheet and the other for growing a thin $(7-8 \mathrm{~cm})$ freshwater ice sheet over a mold with known statistical surface roughness parameters. The saline ice tank, of 25-cm depth and 6-ft diameter, is mounted to its base through a 3-in diameter axle. A pair of flanged bearings support the axle at the base, facilitating rotation of the tank during measurements. The freshwater ice tank, of $15-\mathrm{cm}$ depth and 6-ft diameter, is mobile (via castoring wheels) to permit the heating needed to detach the ice sheet from the mold.

In preparation for ice growth and subsequent measurements, the saline ice tank was placed on wooden beams over the catch basin of the large outdoor pond at CRREL. Freezing of the thick saline ice sheet (from water of $20 \mathrm{ppt}$ salinity) was initiated about three weeks prior to measurements to ensure an ice sheet of large optical thickness. This sheet was allowed to freeze quiescently under essentially the same environmental conditions as the saline ice grown in the adjacent large pond. Growth of the thin freshwater ice sheet was accomplished in just a few days prior to the measurements. In preparing for the growth of this ice sheet the 6-ft diameter mold with statistically known rough surface was placed upright in the tank and held in place (to counteract buoyancy) by means of wooden clamps at each edge. Then freshwater was poured over the mold until the average covering of the mold was about $7 \mathrm{~cm}$. Then the ice sheet was allowed to form quiescently. Note that freshwater was used due to problems encountered in freezing low salinity brine in a confined space, i.e., the mold. That is, as ice forms 


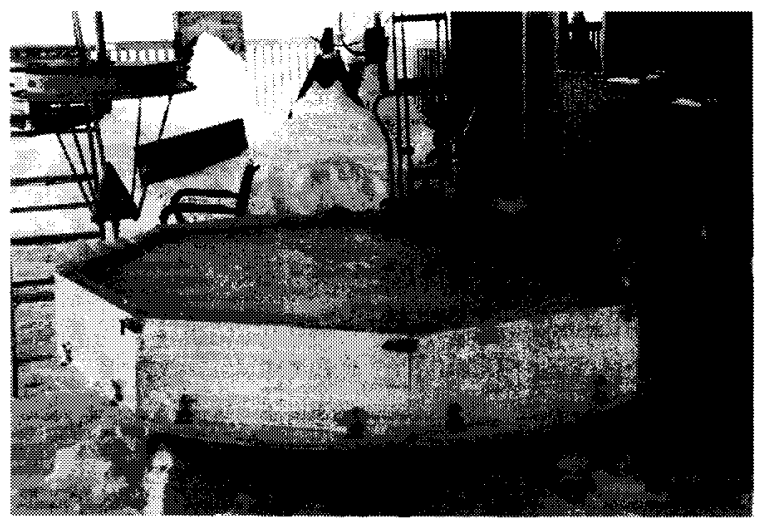

Fig. 1. Photograph of the thick saline ice sheet in the rotating tank.

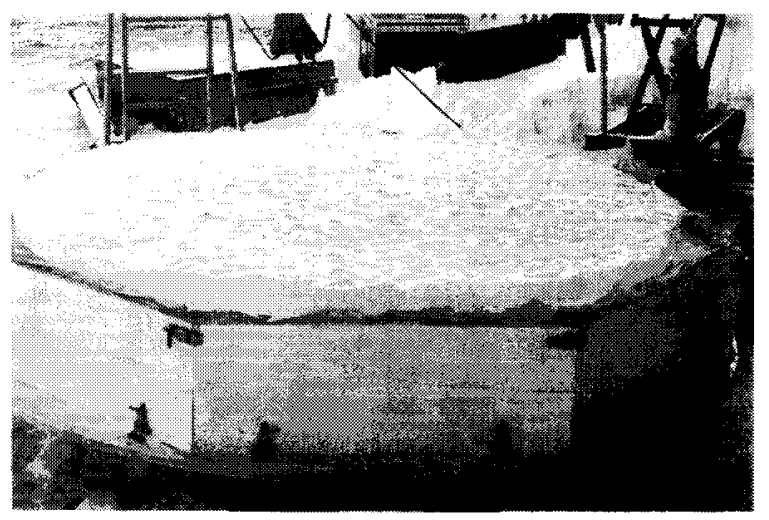

Fig. 2. Photograph of the saline ice with added rough freshwater layer.

from such a solution brine is expelled so that the salinity of the remaining solution continually increases as freezing occurs.

The mold used was selected to have a single-scale Gaussian surface with a standard height deviation of $0.25 \mathrm{~cm}$ and a Gaussian correlation function with correlation length of 2 $\mathrm{cm}$. The target diameter was chosen to be $6 \mathrm{ft}$ to permit most microwave instruments used at CRREL to perform measurements to at least $45^{\circ}$ incidence without interference from the tank edges. The mold was developed by using suitable Monte Carlo simulations and then transferring the data obtained to a computer-controlled milling machine where the simulated surface was implemented in 16-lb polyurethane proofing foam. This procedure is discussed in some detail in [1], [2]. Once the mold surface was milled it was painted with several coats of white latex paint to fill the small air holes present in the foam. Next, the upper surface of the mold was painted with silver particle paint containing $56.3 \%$ silver with a conductive polymer base in order to achieve a good-conducting surface. Hence, this mold can be used as a measurement reference or calibration target as well. Finally, the entire mold was coated with marine epoxy paint to provide protection of the foam and the surface coatings.

The thick saline ice sheet shown in Fig. 1 was measured after it achieved the desired thickness as described in the following section. Roughening of this ice sheet involved removing the rough freshwater ice sheet from the mold and placing it on the top surface as shown in Fig. 2. For reference a mold with the same roughness parameters is shown in Fig. 3. Removing the freshwater ice from the mold and moving it to

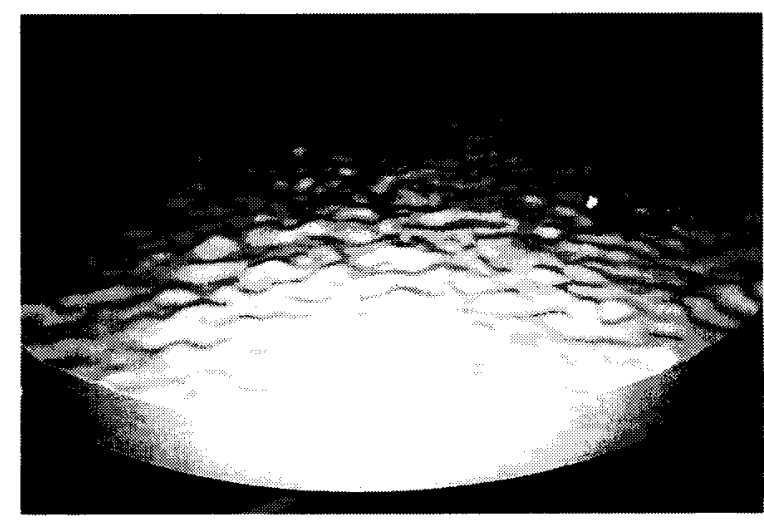

Fig. 3. Photograph of surface mold.

the saline ice tank was a substantial effort. First, the freshwater ice tank was moved into an adjacent heated shed and the sides of the tank were removed. At the $50^{\circ}$ to $60^{\circ} \mathrm{F}$ temperature in the shed approximately $6 \mathrm{~h}$ were needed before the ice sheet reached the freezing point. Once this point was reached the ice sheet was freed from the mold by applying gentle pressure at each of its sides. The ice sheet was then placed on top of the thick saline ice sheet.

\section{DESCRIPTION OF MEASUREMENTS}

The radar instrument used to perform the measurements consists of a HP 8722 Network Analyzer as the transmitter/receiver. This instrument was used in a step-frequency radar mode over $2-18 \mathrm{GHz}$ with 1601 sampling frequencies. The antenna is a 42 -in offset-fed reflector operating in the near field. This antenna, developed by the ElectroScience Laboratory at The Ohio State University, uses a $2-18 \mathrm{GHz}$ AEL horn located at the focal point of the antenna so that the reflected rays are both parallel and in phase across the reflector's aperture [3]. The antenna propagates a uniformly phased plane wave over an area equivalent to the reflector size for ranges up to the ratio of the reflector diameter squared and two wavelengths, i.e., $\frac{D^{2}}{2 \lambda}$ [3]. The near-field measurement technique is superior to the far-field technique with respect to accurately indicating the angular scattering behavior of the target at low incidence angles. When operating in the far field the measurement consists of an average of scattering over the beamwidth of the antenna. As a result the change of target scattering versus angle near nadir is strongly influenced by the antenna pattern. On the other hand, an antenna operating in the near field has a very sharp directional characteristic, i.e., the beamwidth of the antenna is negligible.

Measurements were first performed on the thick bare saline ice sheet. Two days later measurements of the same sheet with the rough freshwater ice sheet laid on top were performed. During both sets of measurements the ambient temperature was less than $0^{\circ} \mathrm{F}$. Data were obtained at $0^{\circ}$ to $50^{\circ}$ incidence, with the bulk of measurements being performed at $10^{\circ}$, $20^{\circ}, 30^{\circ}, 40^{\circ}$, and $50^{\circ}$. For each angle 30 sweeps were obtained as a function of azimuth target position (i.e., azimuth position relative to the radar), where the tank was rotated about $10^{\circ}$ between measurements. This is in accordance with measurements of a surface target with the same roughness 


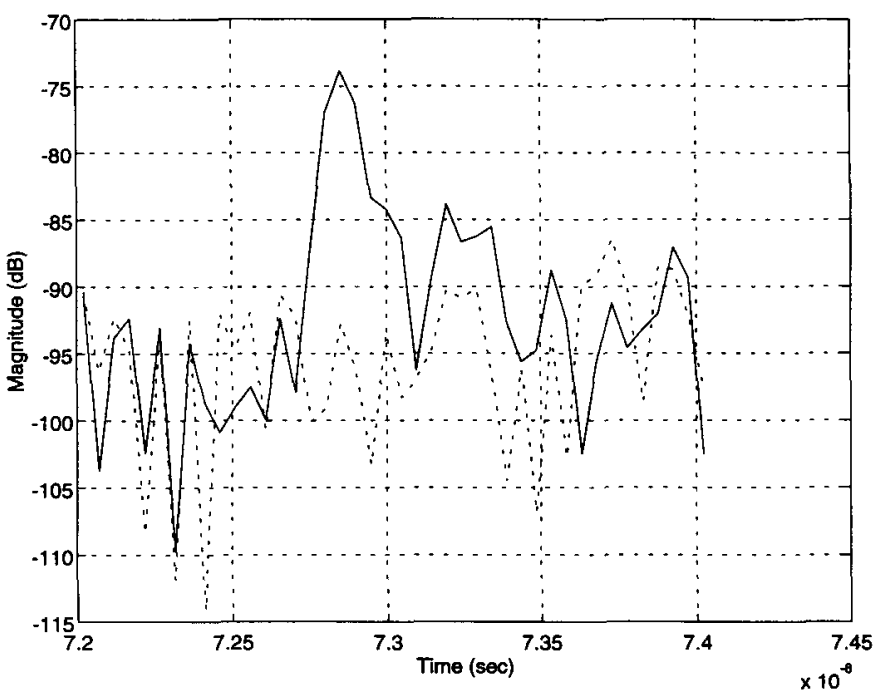

Fig. 4. Time domain responses of 9-in sphere (solid line) and absorber at $20^{\circ}$ incidence.

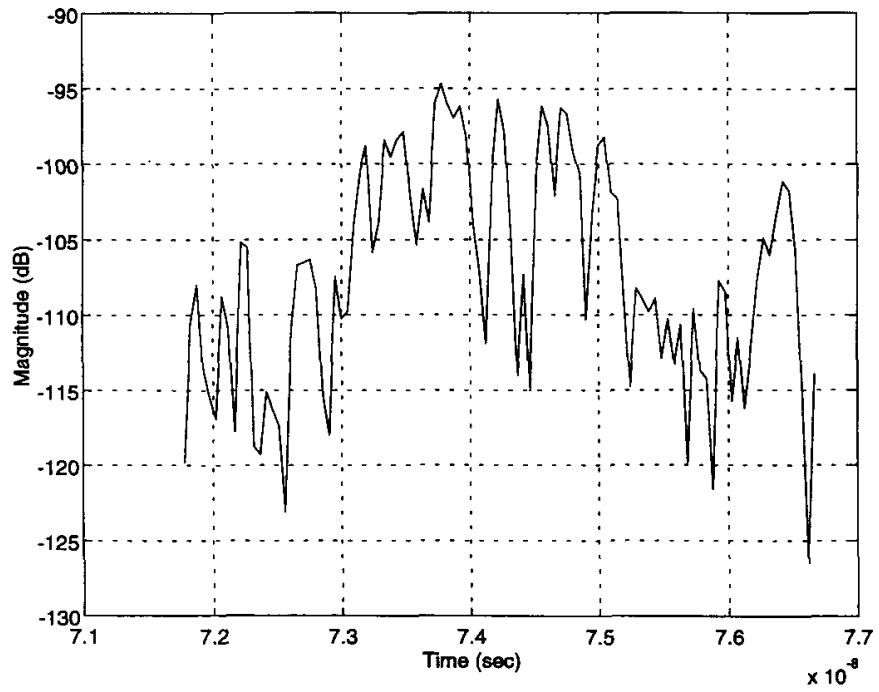

Fig. 5. Time domain response of saline ice target at $20^{\circ}$ incidence.

characteristics at the University of Texas at Arlington that indicated decorrelation after about $5^{\circ}$ change in azimuth. After every 40 or 50 sweeps calibration measurements were obtained using a 9-in diameter metallic sphere which was placed on top of a thin sheet of microwave absorber material, which was placed on top of the ice sheet. The sphere was placed at approximately the midpoint of the antenna beam, and measurements were performed at $20^{\circ}$ incidence to reduce effects of specular scattering from the ice surface. Fig. 4 shows the time domain response of the 9-in sphere and, for comparison, a measurement after the sphere was removed with the absorbing material in place. The peak at approximately $72.8 \mathrm{~ns}$ is the primary reflection from the sphere, while the peak at $73.2 \mathrm{~ns}$ is due to interaction between the sphere and absorber. The secondary (creeping) wave occurs after $74 \mathrm{~ns}$ and is not included in the calibration for reasons discussed in the following section. For comparison, in Fig. 5 is shown the time domain response of the ice surface at $20^{\circ}$ incidence. Here the return occurs between about $73 \mathrm{~ns}$ and $75.5 \mathrm{~ns}$, while the peak at $76.5 \mathrm{~ns}$ is from the tank edge. Processing of the data including calibration are described in the following section.

\section{DATA ANALYSIS AND RESULTS}

The HP 8722 collects data of phase and amplitude as a function of frequency. The time domain plots shown in Figs. 4 and 5 are obtained (by the HP 8722) via Fourier transforming these data. The time domain plots are primarily used to identify components of the return, i.e., returns from the ice sheet, returns from the tank edge, reflections from antenna components, and multiple bounces between the ice sheet and antenna. Once the return from the ice sheet is identified the other returns are eliminated (gated out), using a tapered gate. The data are then Fourier transformed back into phase and amplitude as a function of frequency; these transformed data include predominantly the scattering effects of the ice sheet. The calibration data are processed in the same manner. Then the following expression is applied on a frequency-by-frequency basis in order to obtain estimates of the scattering coefficient for the ice sheet:

$$
\hat{\sigma}^{\circ}(f, \theta)_{\text {ice sheet }}=\frac{P(f, \theta)_{\text {ice sheet }} \cdot \sigma(f)_{\text {theor sphere }}}{P(f)_{\text {sphere }} \cdot A(\theta)_{\text {ant bearn }}}
$$

where

1. $P(f, \theta)_{\text {ice sheet }}$ is the power returned by the ice sheet at frequency $f$ at incidence angle $\theta$,

2. $\sigma(f)_{\text {theor sphere }}$ is the theoretical radar cross section for a perfect conducting sphere at frequency $f$,

3. $P(f)_{\text {sphere }}$ is the power returned by the sphere at frequency $f$,

4. $A(\theta)_{\text {ant beam }}=\frac{\pi r^{2}}{\cos \theta}$ is the area being illuminated on the surface of the ice sheet at an incidence angle of $\theta$ where $r=21$ in for the antenna used, and

5. $\hat{\sigma}^{\circ}(f, \theta)$ ice sheet is the scattering coefficient of the ice sheet at frequency $f$ at incidence angle $\theta$.

It should be noted that $\hat{\sigma}^{\circ}(f, \theta)$ ice sheet is a random variable. Depending on the particular incidence angle one sample of $\hat{\sigma}^{\circ}(f, \theta)_{\text {ice sheet }}$ may vary by up to several $\mathrm{dB}$ from another sample. Hence, for each incidence angle, an average of about 30 samples was obtained via measurements over azimuth and, additionally, averaging (smoothing) over about $300 \mathrm{MHz}$ in bandwidth was done. The net result is the estimate

$$
\hat{\sigma}^{\circ}(f, \theta)_{\text {ice sheet }}=\sum_{f_{i}=f}^{f+\Delta f} \sum_{\text {azimuth }} \hat{\sigma}^{\circ}\left(f_{i}, \theta\right)_{\text {ice sheet }}
$$

that varies by no more than about $\pm 1 \mathrm{~dB}$.

The pattern data for the antenna that were available at the time of the experiments indicated very good uniformity in amplitude over the antenna aperture. However, after additional patterns of the antenna were produced it was discovered that at some frequencies the amplitude at the center of the beam varied by up to several $\mathrm{dB}$ from the amplitude observed elsewhere in the aperture. This was a cause of concern because the calibration target was placed near the center of the antenna beam, whereas the return from the ice sheet is weighted by the pattern of the antenna. We addressed this 


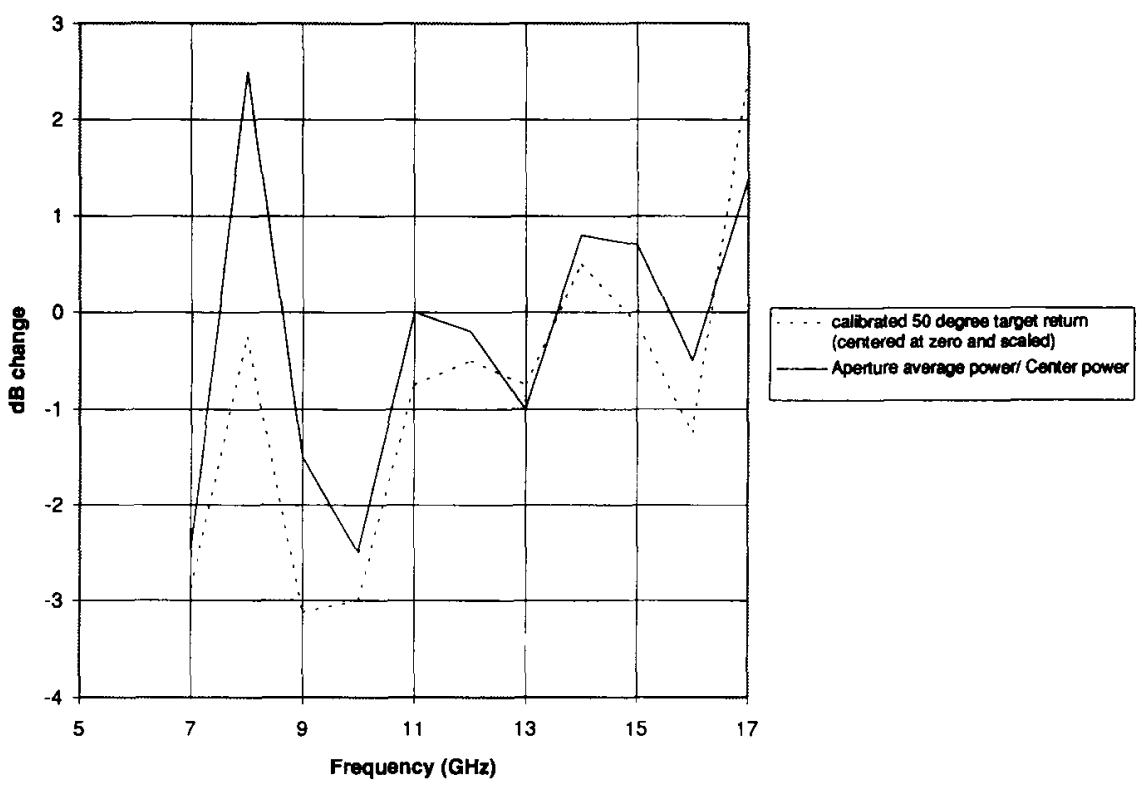

Fig. 6. Behavior of power averaged over the antenna aperture relative to power at center of aperture. Also shown for comparison is the effect on the calibrated data.

by evaluating the available antenna patterns. In this case these were obtained by scanning a measurement probe to measure how the relative power behaves (varies) over the aperture at selected frequencies between $2 \mathrm{GHz}$ and $18 \mathrm{GHz}$; unfortunately, only two linear orthogonal scans were available for each pattern. An average of power over the aperture was obtained for those selected frequencies and compared to the power at the center of the aperture. Only those frequencies where the average aperture power was approximately the same as at the center were further considered. A plot of this ratio of average aperture power to center aperture power is shown in Fig. 6. Note that this curve is discretized to 11 points to illustrate the major features; the complete curve actually crosses 0 near the selected frequencies. To illustrate to what extent this variation affected the data, the variation of the scattering coefficient for the bare ice at $50^{\circ}$ is included. Although the two plots are on different scales, it is clear that the scattering behavior is strongly influenced by the amplitude variations across the aperture of the antenna. Once again, we chose to closely examine only frequencies near the $0 \mathrm{~dB}$ crossings of Fig. 6, i.e., these are the frequencies where the power at the center of the antenna beam is equal, on the average, to the values elsewhere across the aperture.

The bare (smooth) saline ice target was studied at selected frequencies over a range of $7 \mathrm{GHz}$ to $17 \mathrm{GHz}$. Since it had only a slightly rough surface (rms height approximately $0.05 \mathrm{~cm}$ ), backscattering measurements at the larger incidence angles and higher frequencies are expected to be dominated by volume scattering. Therefore, measurements at $50^{\circ}$ are used to determine the albedo at various frequencies. Once measurements were completed on the bare saline ice, the thin rough freshwater ice layer was placed on top of the thicker saline ice sheet. This effectively generated a saline ice sheet with statistically known random roughness.
Because a detailed study of freshwater ice volume scattering effects and interface scattering effects, i.e., between saline and freshwater ice sheets, was not possible, we made substantial efforts to minimize the bubble content of the freshwater ice and to keep both interfaces as planar as possible. It appeared that the ice contained no more than a few percent by volume fraction of air bubbles, and these were predominantly less than $1 \mathrm{~mm}$ in diameter. In comparing measurements with model predictions it appears that volume scattering of the freshwater ice layer became significant for higher frequencies and incidence angles, as described below. We also evaluated the data for evidence of interface scattering effects. At $10^{\circ}$ incidence we were able to observe the primary contribution of the interface due to the high resolution of the radar; this contribution was several $\mathrm{dB}$ lower than the total contribution from the surface. As incidence angle increased, the effect of the interface appeared to decrease even further. Hence, it doesn't appear that the interface was an important contributor in our experiment. When the two surfaces were joined during the experiment the joint seemed to be very tight, i.e., no gap was apparent.

Calibration was done using a 9-in diameter aluminum sphere placed on top of a sheet of microwave absorber over the ice sheet. A gate width of about 1 ns centered near the major peak was applied to the data shown in Fig. 4. This narrow gate was used to keep the effect of noise as low as possible while including the major (primary) scattering of the sphere. It was not possible to include the secondary (creeping) wave component, which occurs at about $75 \mathrm{~ns}$, since it was more than $20 \mathrm{~dB}$ lower than the primary component and since interaction of the sphere with the absorber (see second peak near $73.2 \mathrm{~ns}$ ) altered the behavior of the creeping wave. This gating arrangement should have caused an error no higher than a fraction of $1 \mathrm{~dB}$. 


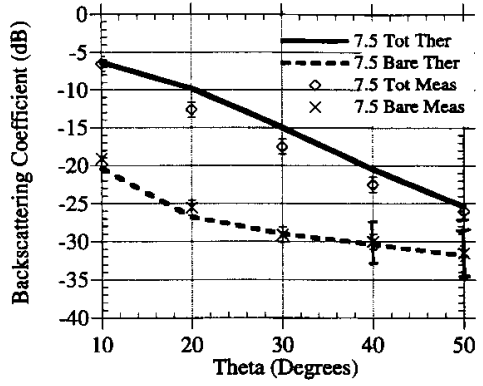

Fig. 7. Measurements versus theory at $7.5 \mathrm{GHz}$. Tot (total) is for ice with added rough surface and Bare is for bare saline ice.

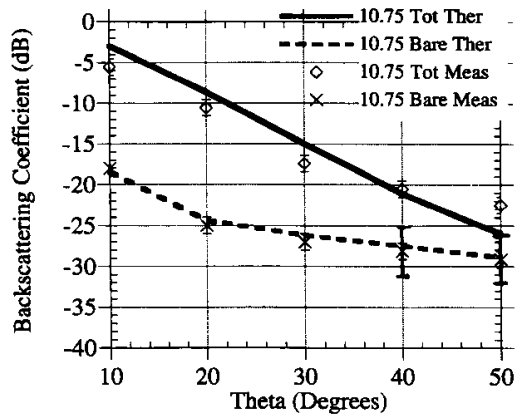

Fig. 8. Measurements versus theory at $10.75 \mathrm{GHz}$. Tot (total) is for ice with added rough surface and Bare is for bare saline ice.

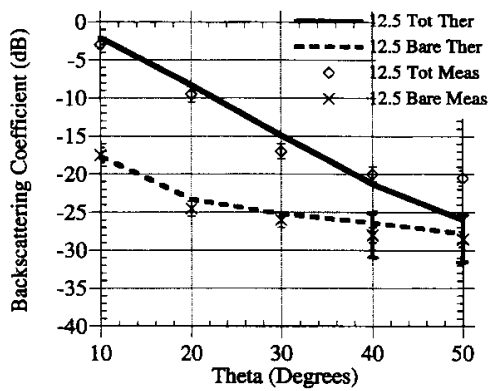

Fig. 9. Measurements versus theory at $12.5 \mathrm{GHz}$. Tot (total) is for ice with added rough surface and Bare is for bare saline ice.

The scattering as a function of incidence angle at $7.5 \mathrm{GHz}$, $10.75 \mathrm{GHz}, 12.5 \mathrm{GHz}, 15.25 \mathrm{GHz}$, and $16.5 \mathrm{GHz}$ for both the bare saline ice and saline ice with rough freshwater ice layer is shown in Figs. 7-11. The error bars show only the uncertainty due to fading effects; an additional uncertainty of about $\pm 1 \mathrm{~dB}$ might be considered reasonable for uncertainty in antenna aperture effects and calibration target gating effects. Notice that larger error bars are given for the higher incidence angle (i.e., $40^{\circ}$ and $50^{\circ}$ ) measurements of the bare saline ice sheet. This is because the signal-to-noise ratio of the measurements was lower for these angles, being only about $6 \mathrm{~dB}$ at the higher frequencies and higher incidence angles.

The parameters used for model comparisons and how they were obtained are shown in Table I. The rms height for the bare saline ice is a typical (average) value for saline ice sheets grown under similar conditions at CRREL (see p. 86 of [4]). The correlation length and albedos were obtained from the best

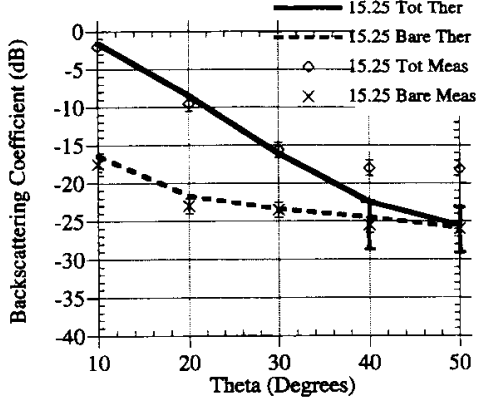

Fig. 10. Measurements versus theory at $15.25 \mathrm{GHz}$. Tot (total) is for ice with added rough surface and Bare is for bare saline ice.

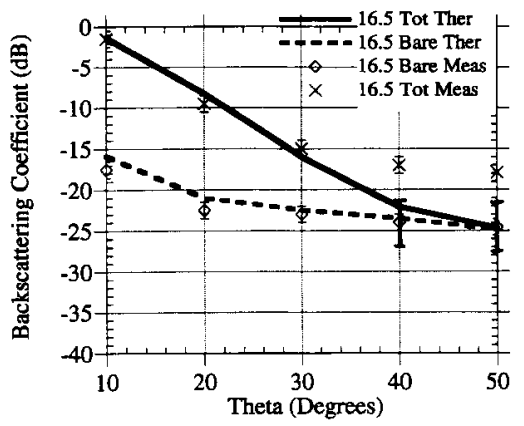

Fig. 11. Measurements versus theory at $16.5 \mathrm{GHz}$. Tot (total) is for ice with added rough surface and Bare is for bare saline ice.

TABLE $\mathbf{I}$

Ground Truth Parameters

\begin{tabular}{l|l|l}
\hline Parameters for model & Bare ice & Rough ice \\
\hline surface rms height & $0.045 \mathrm{~cm}^{*}$ & $0.25 \mathrm{~cm}$ \\
\hline correlation length & $5 \mathrm{~cm}^{* *}$ & $2 \mathrm{~cm}$ \\
\hline correlation function & exponential* & Gaussian \\
\hline albedo at $7.5 \mathrm{GHz}$ & $0.002^{* *}$ & $0.002^{* * *}$ \\
\hline albedo at $10.75 \mathrm{GHz}$ & $0.004^{* *}$ & $0.004^{* * *}$ \\
\hline albedo at $12.5 \mathrm{GHz}$ & $0.0052^{* *}$ & $0.0052^{* * *}$ \\
\hline albedo at $15.25 \mathrm{GHz}$ & $0.008^{* *}$ & $0.008^{* * *}$ \\
\hline albedo at $16.5 \mathrm{GHz}$ & $0.01^{* *}$ & $0.01^{* * *}$ \\
\hline ice sheet thickness & $16 \mathrm{~cm}$ & $9 \mathrm{~cm}$ \\
\hline surface salinity (scraping) & 11 to $28 \mathrm{ppt}$ & \\
\hline underlying brine salinity & $104 \mathrm{ppt}$ & \\
\hline & & \\
\hline * estimated using typical value & & \\
\hline **obtained by fit with model & & \\
\hline ***kept same as for bare & & \\
saline ice & & \\
\hline
\end{tabular}

fit with the multiple-angle, multiple-frequency measurements. Notice that the albedo values are low, and that they show a significant increase with frequency, as expected for saline ice. The optical depth is assumed to be large so that scattering from the bottom of the saline ice can be ignored. This appears to be a very reasonable assumption given the physical thickness and salinity values at the top of the ice sheet and the salinity of the underlying brine (see Table I). The surface roughness for the freshwater ice layer is expected to be identical to that of the mold used to form the ice.

The backscattering from the inhomogeneous medium is approximated as the sum of scattering from the top surface 
and volume scattering from the inhomogeneous medium. The rough top boundary is modeled using the integral equation method (IEM) surface scattering model [5], and its backscattering coefficient is given by

$$
\begin{aligned}
\sigma_{p p}^{\circ} & =4 \pi \cos \theta_{s}\left[I_{p}\left(\mu_{s}, \phi_{s}\right) / I_{p}^{i}\right]_{\theta=\theta_{s}} \\
& =\frac{k^{2}}{2} \exp \left(-2 k_{z}^{2} \sigma^{2}\right) \sum_{n=1}^{\infty}\left|I_{p p}^{n}\right|^{2} \frac{W^{(n)}\left(-2 k_{x}, 0\right)}{n !}
\end{aligned}
$$

where $k_{z}=k \cos \theta, k_{x}=k \sin \theta, p p=v v$ or $h h$,

$$
\begin{aligned}
I_{p p}^{n}= & \left(2 k_{z} \sigma\right)^{n} f_{p p} \exp \left(-k_{z}^{2} \sigma^{2}\right) \\
& +\frac{\left(k_{z} \sigma\right)^{n}\left[F_{p p}\left(-k_{x}, 0\right)+F_{p p}\left(k_{x}, 0\right)\right]}{2}
\end{aligned}
$$

and the symbol $W^{(n)}\left(-2 k_{x}, 0\right)$ is the Fourier transform of the $n$th power of the surface correlation function. Other symbols are given in Appendix 2A of [5]. The thick layer with Rayleigh backscattering from the volume is modeled as

$$
\begin{aligned}
\sigma_{v p p}^{\circ}= & 0.5\left(\kappa_{s} / \kappa_{e}\right) T_{1 t} T_{t 1} \cos \theta\left[1-\exp \left(-2 \kappa_{e} d / \cos \theta_{t}\right)\right] \\
& \times P_{p p}\left(\cos \theta_{t},-\cos \theta_{t} ; \pi\right),
\end{aligned}
$$

where $\kappa_{s} / \kappa_{e}$ is the albedo; $P_{p p}$ is taken to be the Rayleigh phase function; $\kappa_{e} d$ is the optical depth of the layer; $\theta$ and $\theta_{t}$ are the angles of incidence and transmission from the medium above into the layer and $T_{i j}$ is the transmissivity from medium $j$ to $I$. It should be noted that this model has been used successfully to predict backscattering from the good conducting mold surface for incidence angles from $10^{\circ}$ to $60^{\circ}$ and frequencies of $5 \mathrm{GHz}$ to $10 \mathrm{GHz}$ [1], [5].

Measurement results versus predictions of this model for the two cases considered in this study (with input parameters of albedo and surface roughness statistics) are shown in Figs. 7-11. As mentioned above, scattering from the bare saline ice target was modeled using estimated surface roughness, and albedo values and correlation length were adjusted to obtain a match with the data, where the optical depth was assumed to be sufficiently large that bottom boundary effects are negligible. The albedo was then used along with the known statistical parameters of the rough surface to model the total scattering from the saline ice with rough surface. All model input parameters for the scattering model are defined in advance for the rough surface case. Good agreement between measured and predicted data will therefore verify the applicability of the model as well as indicate relative contributions from surface and volume scattering.

Saline ice volume scattering at $7.5 \mathrm{GHz}$ (Fig. 7) is small, as evidenced by the small backscattering coefficient for bare saline ice at $50^{\circ}$. The backscattering from the bare saline ice is dominated by the surface for incidence angles up to less than $20^{\circ}$. Above that the volume scattering component becomes important, and above $40^{\circ}$ it dominates. With the rough layer present, surface scattering dominates for the entire range of incidence angles. Notice that the freshwater ice layer contributed a significant volume scattering effect for higher incidence angles, particularly for frequencies of $12.5 \mathrm{GHz}$ and above $(5 \%$ volume fraction of $1 \mathrm{~mm}$ diameter air bubbles in the freshwater ice layer could have caused this level of effect). As the frequency increases to $X$-band (Fig. 8), the volume scattering component of the saline ice becomes more important. This is observed as an increase in the bare ice scattering level at $50^{\circ}$. The highest incidence angle to which surface scattering dominates also decreases as compared to that for $7.5 \mathrm{GHz}$. With the rough layer present surface scattering dominates to about $40^{\circ}$ and the volume scattering becomes noticeable at $50^{\circ}$. By careful study of both the bare saline ice scattering and saline ice with added rough surface (Figs. 7-12) it becomes apparent that some general observations can be made from the data. The volume scattering component becomes more important as frequency increases; this is observed as an increase in the bare ice scattering level at $50^{\circ}$. Its impact is also seen by locating (on the bare ice scattering curves) the highest incidence angle for which surface scattering dominates; this decreases as frequency increases. Once the rough layer is added the effect of surface scattering becomes more important. However, even with the rough layer saline ice volume scattering becomes more important and, in fact, dominates for the higher incidence angles and highest two frequencies, i.e., $15.25 \mathrm{GHz}$ and $16.5 \mathrm{GHz}$. This is, in part, due to the fact that the molded rough surface has a Gaussian correlation function. Once again, the results are summarized in Fig. 12.

A few additional comments concerning the results are in order. First, notice that the measurements of the rough surface target agree quite well with model predictions, except as frequency increases measurements and predictions begin to deviate, first at $50^{\circ}$ incidence and then also at $40^{\circ}$ incidence. This appears to be due to a volume scattering component of the added rough surface layer; a simple consideration of Rayleigh scattering for a 5\% volume fraction of $1 \mathrm{~mm}$ diameter air bubbles indicates this level of volume scattering. The frequency and angular regions over which good agreement was found provide confidence in the model predictions. The model predictions are then used to assess what the relative contributions of volume and surface scattering would be for a rough saline ice sheet, excluding the air bubble contributions. Second, these results are for a surface which has a Gaussian correlation; for a surface of the same rms height but exponential correlation the significance of surface scattering would be expected to extend to higher incidence angles.

\section{SUMMARY}

The relative contributions of surface and volume scattering were determined for a saline ice sheet with well-known surface roughness characteristics. This was accomplished via controlling surface roughness characteristics, using a sensitive near-field measurement instrument, and comparing measurements with model predictions using combined surface and volume scattering models to predict total scattering. The results show that volume can be important (dominant) at higher angles of incidence for very slightly rough surfaces and for Gaussian correlated surfaces. The relative importance of volume and surface scattering depends greatly on frequency, incidence angle, volume scattering albedo, surface roughness, and surface correlation function. The successful comparison 


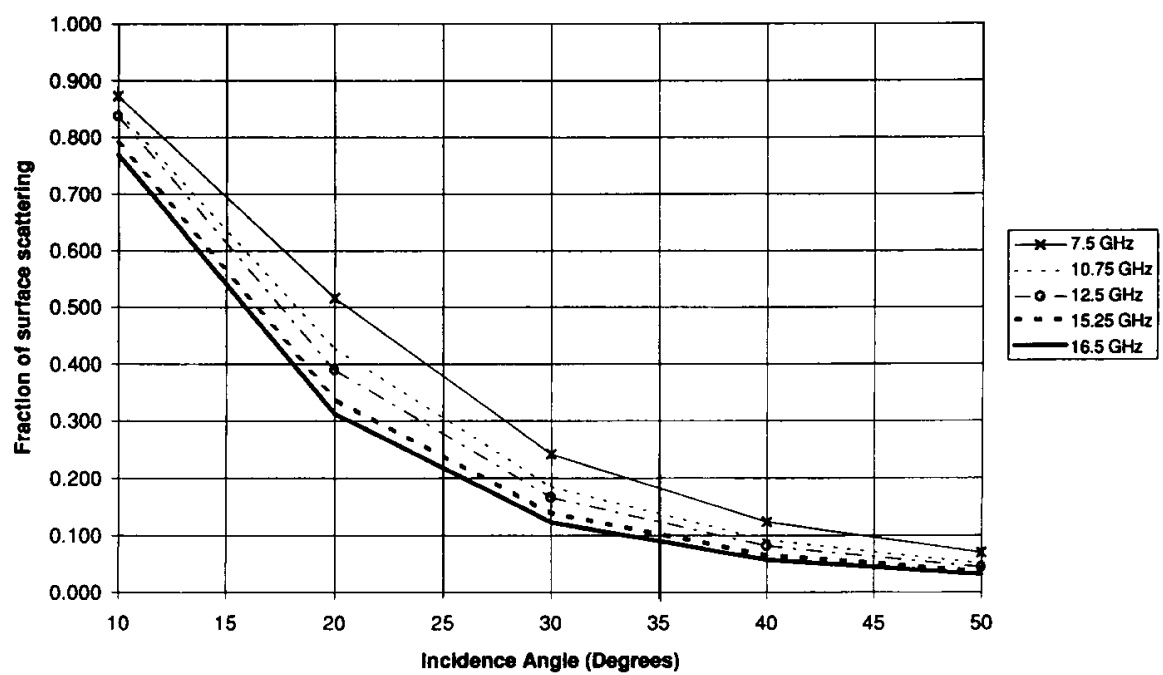

(a)

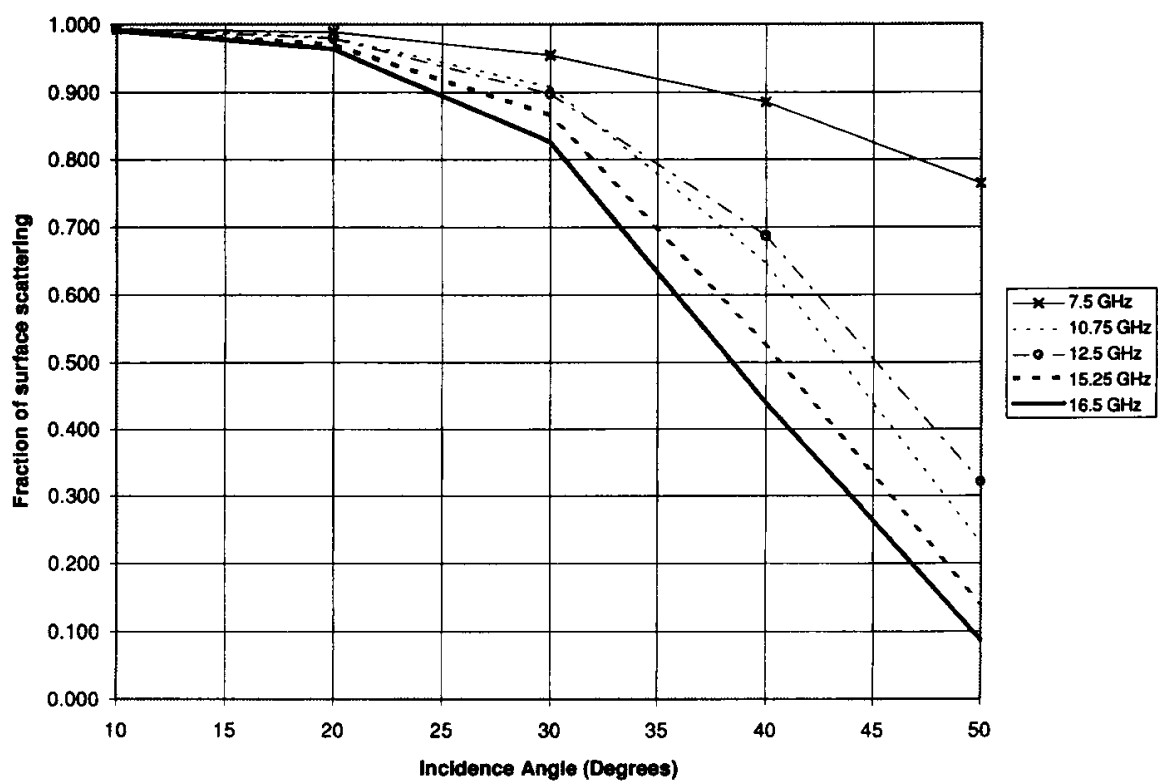

(b)

Fig. 12. (a) Percent contribution due to surface scattering for bare saline ice, (b) Percent contribution due to surface scattering for saline ice with rough freshwater layer.

between the scattering model and measurements at small angles is due to use of a plane wave antenna which eliminated concern about antenna angular resolution.

The results imply that surface scattering is always important for first-year ice at low incidence angles. It is also important at higher incidence angles for the first-year ice roughnesses most often found in the field, i.e., $0.1-0.2 \mathrm{~cm} \mathrm{rms} \mathrm{height.}$ The importance of the volume scattering contribution varies considerably: The contribution may be very small for rougher first-year ice $(0.4-0.5 \mathrm{~cm} \mathrm{rms}$ height), or it may dominate at higher incidence angles for ice formed under quiet conditions, i.e., smooth ice such as may be found in leads (approximate rms height of $0.05 \mathrm{~cm}$ ).

Other experiments using these ice tanks, mold, and measurement instruments are being planned. These will place emphasis on determining surface and volume scattering contributions for snow-covered ice, desalinated ice, studying statistics of the scattering, and assessing polarimeteric behaviors. In addition we anticipate using the mold, which has a good-conducting rough surface, as a calibration target that will account for nonuniformities of the antenna beam amplitude.

\section{ACKNOWLEDGMENT}

The authors thank J. Govoni and B. Elder of CRREL for their help in growing the ice sheets and in maintaining the experimental setups.

\section{REFERENCES}

[1] C. Nance, "Scattering and image analysis of conducting rough surfaces," Ph.D. dissertation, Dep. Elect. Eng., Univ. of Texas, Arlington, 1992. 
[2] J. Rochier, A. Blanchard, and M. Chen, "The generation of surface targets with specified surface statistics," Int. J. Remote Sensing, vol. 10, no. 7, pp. 1155-1174, 1989

[3] K. Jezek et al., "Microwave scattering from saline ice using plane wave illumination," in IGARSS '94 Proc., pp. 493-495.

[4] F. D. Carsey, Ed., Microwave Remote Sensing of Sea Ice. Washington, DC: American Geophysical Union, 1992, Geophysical Monograph 68.

[5] A. K. Fung, Microwave Scattering and Emission Models and Their Applications. Norwood, MA: Artech House, 1994.

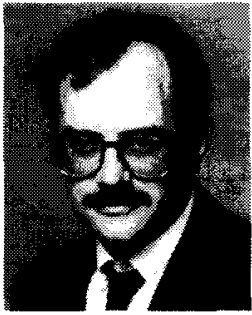

Jonathan W. Bredow (S'85-M'89) received the B.S.E.E. degree from Kansas State University, Manhattan, in 1977, the M.S.B.M.E. degree from Iowa State University, Ames, in 1980, and the Ph.D degree from the University of Kansas, Lawrence, in 1989.

Since 1989 he has been on the faculty of the Department of Electrical Engineering at the University of Texas at Arlington (UTA), where he is now an Associate Professor. His research interests include radar systems, RCS measurements of discrete and distributed targets, antennas, and microwave circuits and systems. He currently oversees activities in the UTA microwave anechoic chamber. He has authored and coauthored a number of papers on scattering from volcanic ash, simulated sea ice, man-made surface, and dense volume media, and inverse SAR imaging of a variety of targets.

Ronald L. Porco was born in Bronx, NY, on June 8, 1967. He received the B.S.E.E. degree from the University of Miami, Coral Gables, FL, in 1989 and the M.S.E.E. and Ph.D. degrees from the University of Texas at Arlington (UTA) in 1991 and 1994, respectively

During his M.S. and Ph.D. programs he served as a Graduate Research Assistant and later as a Research Associate at the UTA Wave Scattering Research Center. His research interests have been in the areas of scattering from dense media, microwave imaging, and remote sensing. He currently works in the Advanced Messaging Systems Division of Motorola, Fort Worth, TX.

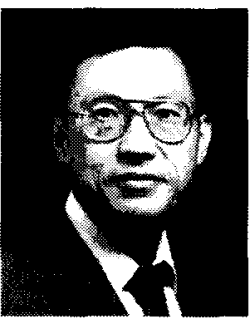

Adrian K. Fung (S'60-M'66-SM'70-F'85) received the B.S.E.E. degree from the Taiwan Provincial Cheng Kung University in 1958, the M.S.E.E. degree from Brown University, Providence, RI, in 1961, and the Ph.D. degree from the University of Kansas, Lawrence, in 1965

He was an EE faculty member at the University of Kansas from 1965 to 1984 , and became a Full Professor in 1972. He has been a Professor of Electrical Engineering at the University of Texas, Arlington, since 1984 and is Director of the Wave Scattering Research Center. His current interests include wave scattering and emission from irregular surfaces and random media, radar image simulation, numerical simulation of radar scattering, and random analysis. He is the author of Microwave Scattering and Emission Models and Their Applications and coauthor of a three-volume book on Microwave Remote Sensing.

Dr. Fung received the 1987 Halliburton Excellence in Research Award, the 1989 Distinguished Research Award from the University of Texas, and the 1989 Distinguished Achievement Award from the IEEE Geoscience and Remote Sensing Society. He is a member of Sigma Xi and the U.S. Commission $\mathrm{F}$ of the International Scientific Radio Union.

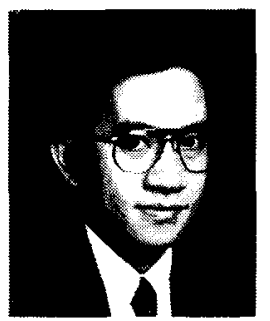

Saibun Tjuatja (S'85-M'93) was born in Medan, Indonesia, on November 6,1962 . He received the B.S.E.E. degree with high honors from the University of Texas, Arlington (UTA), in 1987, the M.S.E.E. degree from Purdue University, West Lafayette, IN, in 1988, and the Ph.D. degree in electrical engineering from UTA in 1992.

From 1992 to 1993 he was a Research Associate at the UTA Wave Scattering Research Center. Since Fall 1993 he has been an Assistant Professor in the Department of Electrical Engineering at UTA. His research interests have been in the areas of remote sensing of the environment, wireless communications, wave scattering and emission from earth terrain, numerical techniques for electromagnetics, and pattern recognition.

Dr. Tjuatja is a member of Tau Beta Pi and Alpha Chi.

Kenneth C. Jezek received the Ph.D. degree in geophysics from the University of Wisconsin, Madison, in 1980.

From 1983 to 1989 , he worked as a Geophysicist with the USA Cold Regions Research and Engineering Laboratory. In 1989, he was appointed Director of the Byrd Polar Research Center and Associate Professor in the Department of Geological Sciences at The Ohio State University. His present research interests include the electromagnetic properties of snow, ice, and frozen ground. He is especially interested in applying spaceborne remote sensing data to problems associated with the growth, motion, and decay of Earth's ice cover.

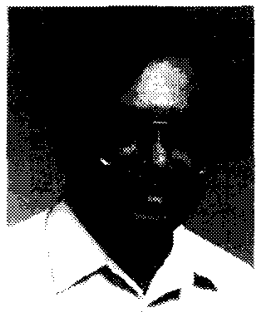

Sivaprasad Gogineni received the Ph.D. degree from The University of Kansas, Lawrence.

$\mathrm{He}$ is currently a Professor in the Department of Electrical Engineering and Computer Science at the University of Kansas and Director of the Radar Systems and Remote Sensing Laboratory. He has been involved in research on the application of radars to the remote sensing of sea ice, ocean, and land. He was actively involved in developing instrumentation for radar systems currently being used at The University of Kansas for backscattering measurements. He has also participated in field experiments in the Arctic and on towers in the open ocean. He has authored or been a coauthor of over 30 journal publications and many technical reports and conference presentations.

Dr. Gogineni is a member of URSI Commission F and the Electromagnetics Academy. In 1991, he was awarded the Miller Award for Engineering Research from the University of Kansas and the Taylor and Francis 1991 Best Letter Award.

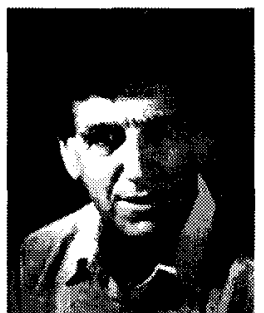

Anthony J. Gow received the M.Sc. degree in geology in 1965 and the D.Sc. degree in geology in 1975 from Victoria University of Wellington, New Zealand.

He is currently employed as a Geologist with the U.S. Army Cold Regions Research and Engineering Laboratory in Hanover, NH. His long-term interests have included studies of the structural, paleoclimate, and physical properties of Antarctic snow and ice. Most recently, he was a member of the US research team investigating the paleoclimate implications of an ice core that penetrated $3053 \mathrm{~m}$ to bedrock in Central Greenland. He has also been involved for a number of years in studies of the crystalline structure and physical properties of lake, river, and sea ice and has extended these studies to laboratory-grown model ices. Since 1985 he has been Adjunct Professor of Glaciology in the Earth Sciences Department at the University of New Hampshire.

Dr. Gow is a member of the American Geophysical Union, the International Glaciological Society, and the Antarctica Society. 